УДК 536.24:533

\title{
ХАРАКТЕРИСТИКИ ЦИРКУЛЯЦИОННЫХ ТЕЧЕНИЙ В БЛИЖНЕМ СЛЕДЕ ЦИЛИНДРИЧЕСКИХ СТАБИЛИЗАТОРОВ ПЛАМЕНИ
}

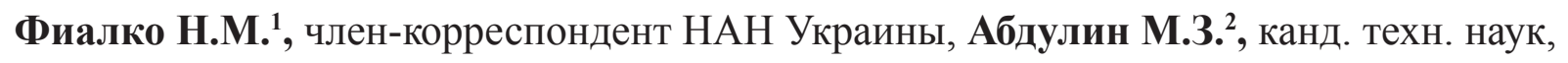
Шеренковский Ю.В. ${ }^{1}$, канд. техн. наук, Майсон Н.В. ${ }^{1}$

${ }^{1}$ Институт технической теплофизики НАН Украины, ул. Желябова, 2а, Киев, 03680, Украина ${ }^{2}$ Национальный технический университет Украины «КПИ», пр. Победы, 37, Киев, 03056, Украина

Представлено результати експериментальних досліджень основних характеристик зон зворотних токів в
закормових областях циліндричних стабілізаторів полум'я. Наводяться дані щодо закономірностей впливу на температурний режим та розміри
цих зон таких факторів, як відносний крок розташування газоподавальних отворів, швидкість повітряного потоку та коефіцієнт надлишку повітря.
Представлены результаты экспериментальных исследований основных характеристик зон обратных токов в закормовых областях цилин-
дрических стабилизаторов пламени. Приводятся данные о закономерностях влияния на температурный
режим и размеры этих зон таких факторов, как относительный шаг расположения газоподающих отверстий, скорость воздушного потока и коэффициент избытка воздуха.

Библ. 3, табл. 2, рис. 2.

Ключевые слова: цилиндрический стабилизатор пламени, зона обратных токов, температурный режим.

$D$ - внутренний диаметр круглого канала;

$d$ - диаметр газоподающих отверстий;

$d_{\text {ст }}$ - внешний диаметр стабилизатора;

$L_{\text {от }}^{\text {ст }}-$ протяженность зоны обратных токов;

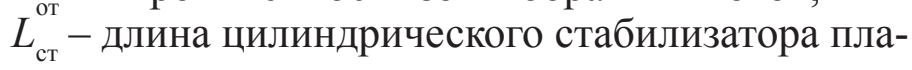
мени;

$L_{1}-$ расстояние от газоподающих отверстий до срывной кромки стабилизатора;

$S$ - шаг расположения газоподающих отверстий;

\section{Введение}

В энергетическом оборудовании различного назначения широко применяются горелочные устройства относительно малой мощности (до 0,2 МВт). Использование таких горелочных устройств оказывается эффективным для энергетического оборудования как сравнительно небольшой, так и различной тепловой мощности в ситуациях, отвечающих высоким требованиям к равномерности теплоподвода по огневому пространству.

В указанных условиях представляется целесо- $t_{\max }$ - максимальная температура в зоне обратных токов;

$t_{\min }-$ минимальная температура в зоне обратных токов;

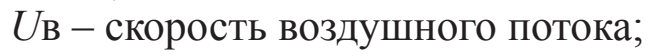

$\alpha$ - коэффициент избытка воздуха;

$\delta_{0}$ - толщина стенок стабилизатора;

$\delta_{1}$ - толщина стенок канала.

образным применение горелок с цилиндрическими стабилизаторами пламени. Данные горелочные устройства характеризуются более высокой эффективностью, чем широко используемые горелки с плоскими стабилизаторами пламени. Это связано прежде всего с отсутствием различного рода потерь, обусловленных краевыми эффектами. Кроме того к важным достоинствам цилиндрических горелочных устройств относятся также простота их интегрирования в конструкцию энергетических установок [1-3].

Применение цилиндрических тел в качестве 
стабилизаторов пламени является одним из распространенных способов реализации устойчивого процесса горения топлива (см. например [4-6]). В связи с потребностями практики проектирования цилиндрических горелочных устройств, в которых используется указанный способ стабилизации пламени, необходимым является проведение специальных исследований процессов переноса в ближнем следе за продольно обтекаемым цилиндром.

\section{Постановка задачи и методика исследований}

Данная работа посвящена экспериментальным исследованиям различных характеристик зон обратных токов в ближнем следе цилиндрических стабилизаторов пламени. При этом особое внимание уделялось определению размеров данных зон и их температурных режимов. Рассмотрению подлежал горелочный модуль, представляющий собой круглый канал с размещенным в нем цилиндрическим стабилизатором со скругленной передней и затупленной задней кромкой (рис. 1). Стабилизатор был снабжен системой круглых отверстий, через которые осуществлялась поперечная подача газа в сносящий поток воздуха. Исследовалась ситуация, отвечающая сжиганию пропана-бутана.
Основные геометрические характеристики рассматриваемого горелочного модуля следующие: $L_{\text {ст }}=200 \cdot 10^{-3} \mathrm{M} ; L_{1}=13,5 \cdot 10^{-3} \mathrm{M} ; D=53 \cdot 10^{-3} \mathrm{M} ; d_{\text {ст }}=$ $=33 \cdot 10^{-3} \mathrm{M} ; \delta_{0}=\delta_{1}=2,5 \cdot 10^{-3} \mathrm{M} ; d=2 \cdot 10^{-3} \mathrm{M}$.

В ходе экспериментов варьировались такие величины: относительный шаг расположения газоподающих отверстий $S / d-$ от 3,2 до 5,0; скорость воздушного потока $U_{\text {в }}$ от 5 до 10 м/с и коэффициент избытка воздуха $\alpha$ - от 2,24 до 6,72 .

При проведении экспериментальных исследований определение размеров зон обратных токов в закормовой области стабилизаторов осуществлялось методом визуализации течения с использованием солей натрия. Температуры в зоне обратных токов измерялись хромель-алюмелевой термопарой с диаметром королька $1,5 \cdot 10^{-3} \mathrm{M}$.

\section{Результаты исследований и их анализ}

Характеристики вариантов выполненных экспериментальных исследований приведены в табл. 1. Основные результаты данных исследований представлены в табл. 2 и на рис. 2. Здесь даны материалы, касающиеся зон обратных токов в ближнем следе стабилизаторов, а именно, их конфигурации, размеров и температурных режимов.

Табл. 1. Характеристики вариантов экспериментальных исследований

\begin{tabular}{|c|c|c|c|c|c|c|c|c|}
\hline № варианта & 1 & 2 & 3 & 4 & 5 & 6 & 7 & 8 \\
\hline$S / d$ & 3,2 & 5,0 & 3,2 & 5,0 & 3,2 & 5,0 & 3,2 & 5,0 \\
\hline$\alpha$ & 2,24 & 2,24 & 6,72 & 6,72 & 2,24 & 2,24 & 6,72 & 6,72 \\
\hline$U_{\text {в }}, \mathrm{M} / \mathrm{c}$ & 5 & 5 & 5 & 5 & 10 & 10 & 10 & 10 \\
\hline
\end{tabular}

Табл. 2. Значения характерных перепадов температуры $\left({ }^{\circ} \mathrm{C}\right)$ в зонах обратных токов за цилиндрическим стабилизатором пламени для различных вариантов экспериментальных исследований

\begin{tabular}{|c|c|c|c|c|c|c|c|c|}
\hline № варианта & 1 & 2 & 3 & 4 & 5 & 6 & 7 & 8 \\
\hline$\Delta t_{31}=t_{3}-t_{1}$ & 440 & 490 & 290 & 320 & 400 & 460 & 330 & 360 \\
\hline$\Delta t_{34}=t_{3}-t_{4}$ & 420 & 380 & 250 & 230 & 350 & 380 & 290 & 330 \\
\hline$\Delta t_{21}=t_{2}-t_{1}$ & 330 & 400 & 190 & 300 & 240 & 340 & 290 & 230 \\
\hline$\Delta t_{32}=t_{3}-t_{2}$ & 110 & 90 & 100 & 20 & 160 & 120 & 40 & 130 \\
\hline$\Delta t_{54}=t_{5}-t_{4}$ & 250 & 250 & 200 & 130 & 180 & 210 & 260 & 200 \\
\hline$\Delta t_{35}=t_{3}-t_{5}$ & 170 & 130 & 50 & 100 & 170 & 170 & 30 & 130 \\
\hline$\Delta t_{41}=t_{4}-t_{1}$ & 20 & 110 & 40 & 90 & 50 & 80 & 40 & 30 \\
\hline$\Delta t_{52}=t_{5}-t_{2}$ & -60 & -40 & 50 & -80 & -10 & -50 & 10 & 0 \\
\hline
\end{tabular}




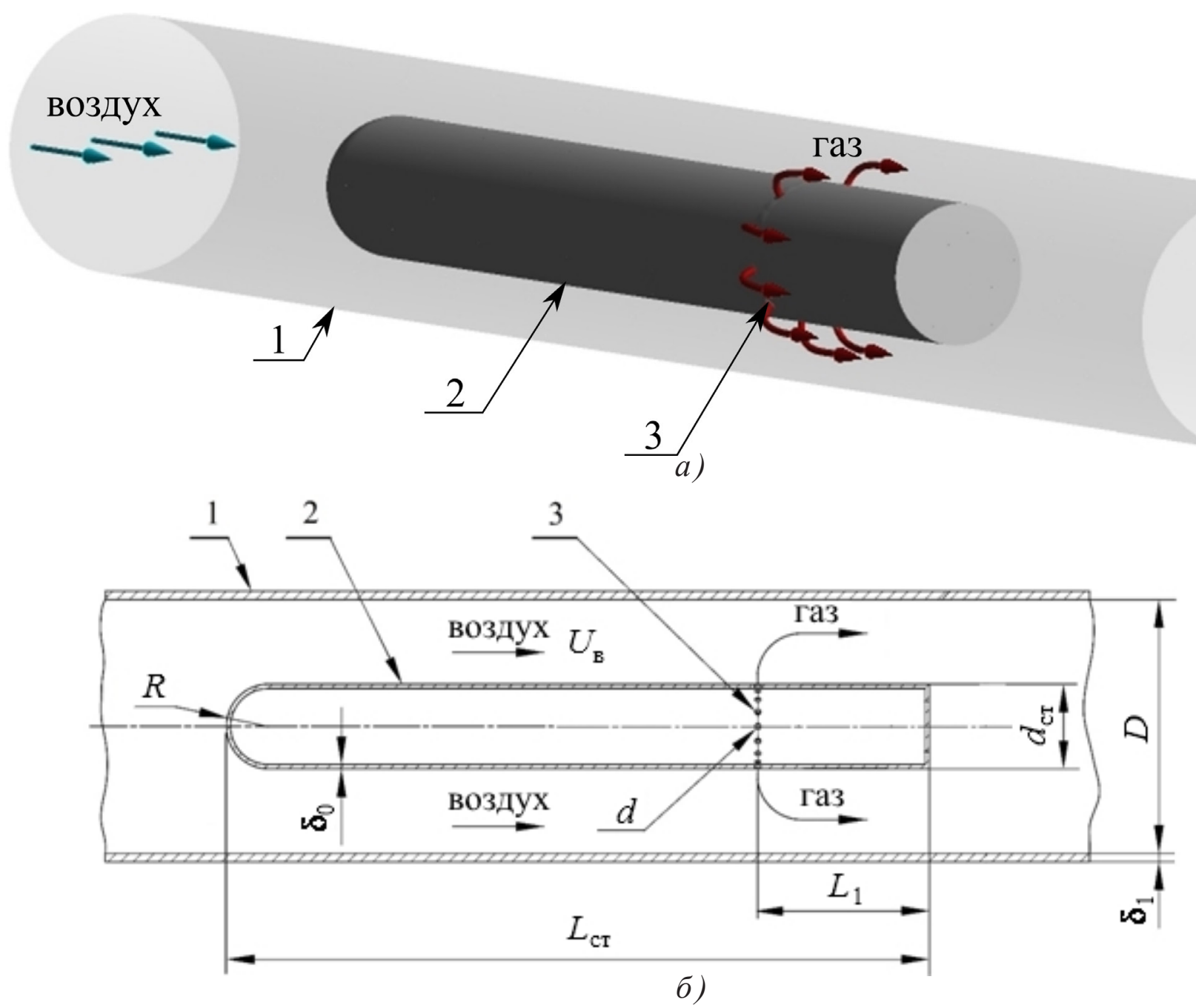

\section{Рис. 1. Схема (а) и продольный разрез (б) горелочного устройства \\ с цилиндрическим стабилизатором пламени: 1 - круглый канал; 2 - цилиндрический стабилизатор пламени; 3-газоподающие отверстия.}

Рассмотрим вначале эффекты влияния варьируемых в ходе экспериментов параметров на одну из важнейших характеристик зоны обратных токов - ее протяженность $L_{\text {от }}$ Что касается шага расположения газоподающих отверстий $S / d$, то как следует из данных, представленных на рис. 2., с его увеличением протяженности зон обратных токов уменьшаются. Так, при $\alpha=6,72$ и $U_{\text {в }}=5 \mathrm{~m} / \mathrm{c}$ значение $L_{\text {от }}$ сокращается с $38 \cdot 10^{-3}$ м до $29 \cdot 10^{-3} \mathrm{M}$ при повышении $S / d$ от 3,2 до 5,0. Отмеченный характер влияния шага $S / d$ обусловлен тем, что с его увеличением возрастает инжектирующее действие струй газа, то есть вовлечение окружающего воздуха в струи. Последнее приводит к дополнительному повышению степени разряжения в зоне обратных токов в закормовой области стабилизатора и соответственно к сокращению ее длины $L_{\text {от }}$.

Обращает на себя внимание также тот факт, что существенное влияние $S / d$ на величину $L_{\text {от }}$ имеет место лишь при относительно больших значениях $\alpha$. Как видно из рис. $2 \partial$, е, ж, з, с увеличением $S / d$ от 3,2 до 5,0 значение $L_{\text {от }}$ уменьшается на $2 \cdot 10^{-3}$ м при $\alpha=2,24$ и на $10 \cdot 10^{-3}$ м при $\alpha=6,72$.

Согласно полученным данным имеет место тенденция к уменьшению протяженности зон обратных токов в ближнем следе стабилизатора при возрастании коэффициента избытка воздуха $\alpha$. При этом, чем больше величина $S / d$ и скорость потока воздуха $U_{\text {в }}$, тем ярче проявляется данная тенденция. Например, для $S / d=5,0$ с увеличением $\alpha$ 
от 2,24 до 6,72 длина зоны обратных токов уменьшается на $6 \cdot 10^{-3}$ м при $U_{\text {в }}=5 \mathrm{M} / \mathrm{c}$ и на $10 \cdot 10^{-3} \mathrm{M}$ при $U_{\text {в }}=10 \mathrm{M} / \mathrm{c}$.

Результаты проведенных исследований свидетельствуют также о том, что повышение скорости

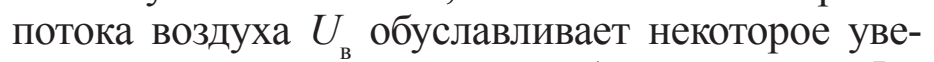
личение протяженности зон обратных токов $L_{\text {от }}$. Это увеличение оказывается более заметным при меньших значениях $\alpha$.

Перейдем далее к анализу температурных режимов зон обратных токов в закормовых областях стабилизаторов. Согласно полученным данным во всем исследуемом диапазоне изменения рассматриваемых параметров наименьшие значения температуры tmin в зоне обратных токов имеют место вблизи срывной кромки стабилизатора, в подобласти, отвечающей началу процесса горения. Максимальные значения температуры $t_{\max }$ в данной зоне наблюдаются в наиболее удаленной от стабилизатора точке 3 на ее границе, расположенной на оси следа (рис. 2).

Что касается влияния шага $S / d$ на значения наибольшей и наименьшей температуры в зоне обратных токов, то, как показали проведенные исследования, с ростом $S / d$ при прочих равных условиях данные температуры повышаются (сопоставьте результаты на рис. $2, a$ и $\sigma, \varepsilon$ и г, $\partial$ и $е$, ж и з). Это, по-видимому, в большой мере связано с отмеченным выше сокращением размеров зон обратных токов, и как следствие, с повышением их теплонапряженности.

Аналогичным оказывается и характер влияния скорости потока воздуха $U_{\text {в }}$ на уровни температур $t_{\min }$ и $t_{\max }$. Здесь повышение указанных температур при возрастании скорости $U_{\text {в }}$ обусловлено в большой степени дополнительной турбулизацией потока в циркуляционных зонах. При этом отмеченное влияние является более существенным при больших значениях коэффициента избытка воздуха. Например, для $S / d=5,0$ при повышении $U_{\text {в } о \text { от } 5}$ до $10 \mathrm{M} / \mathrm{c}$ возрастание температуры $t_{\max }$ составляет $30{ }^{\circ} \mathrm{C}$ и $130{ }^{\circ} \mathrm{C}$ для $\alpha=2,24$ и $\alpha=6,72$ соответственно.

Полученные данные свидетельствуют также о том, что максимальные и минимальные температуры в зонах обратных токов закормовых областей стабилизаторов существенно зависят от коэффициента избытка воздуха $\alpha$. С ростом $\alpha$ уровни $t_{\max }$ и $t_{\min }$ заметно снижаются. Причем это снижение для $t_{\max }$ оказывается более значительным при меньших величинах скорости потока воздуха $U_{\text {в }}$ Так, при $S / d=5,0$ повышение $\alpha$ от 2,24 до 6,72 приводит к падению $t_{\max }$ на $140{ }^{\circ} \mathrm{C}$ при $U_{\text {в }}=10 \mathrm{~m} / \mathrm{c}$, и на $240^{\circ} \mathrm{C}$ при $U_{\text {в }}=5 \mathrm{M} / \mathrm{c}$.

Относительно характера изменения температур в зоне обратных токов за стабилизатором в первую очередь следует отметить, что с удалением от торцевой поверхности стабилизатора уровни температур в данной зоне повышаются. При этом данное повышение может быть весьма существенным. Например, вдоль границы зоны обратных токов 1 - 3 перепад температур $\Delta t_{31}$ достигает $490{ }^{\circ} \mathrm{C}$ для ситуации, отвечающей $S / d \stackrel{31}{=} 5,0, \alpha=2,24$ и $U_{\text {в }}$ $=5 \mathrm{M} / \mathrm{c}$ (вариант исследований № 2). Важно подчеркнуть, что указанная тенденция к повышению температуры при удалении от торцевой поверхности стабилизатора наиболее заметно проявляется вблизи данной поверхности и становится менее ярко выраженной в хвостовой части зоны обратных токов. Так, для варианта исследования № 2, перепад температур $\Delta t_{21}$ на участке $1-2$ границы зоны обратных токов, примыкающем к торцу стабилизатора, составляет $400{ }^{\circ} \mathrm{C}$, а на участке $3-2$, отвечающем хвостовой части зоны обратных токов, - лишь $90{ }^{\circ} \mathrm{C}$. Как видно из табл. 2, данная тенденция сохраняется также и для характера изменения температуры вдоль оси следа (сопоставьте перепады температур $\Delta t_{54}$ и $\Delta t_{35}$ ).

Что касается картины изменения температур в зоне обратных токов в поперечном направлении, то здесь уровни температур меняются значительно менее существенно, чем по длине этой зоны. Согласно полученным данным для варианта исследования № 6 перепад температур $\Delta t_{34}$ в зоне обратных токов вдоль оси следа равняется $380{ }^{\circ} \mathrm{C}$, а соответствующий перепад $\Delta t_{41}$ на половине ширины зоны вблизи торца стабилизатора составляет лишь $80^{\circ} \mathrm{C}$.

Как показали результаты проведенных исследований, перепад температур $\Delta t_{41}$ в поперечном направлении вблизи торцевой поверхности стабилизатора во всех рассмотренных ситуациях оказывался положительным. То есть температура внутри зоны обратных токов $t_{4}$ на оси следа превышает соответствующие значения температуры на границе этой зоны $t_{1}$ вблизи срывной кромки 


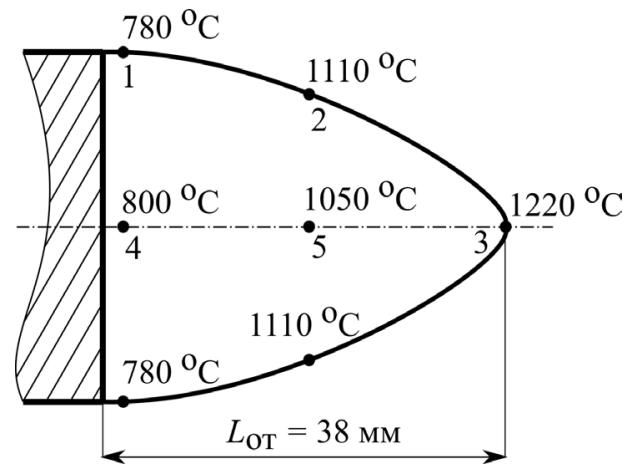

a)
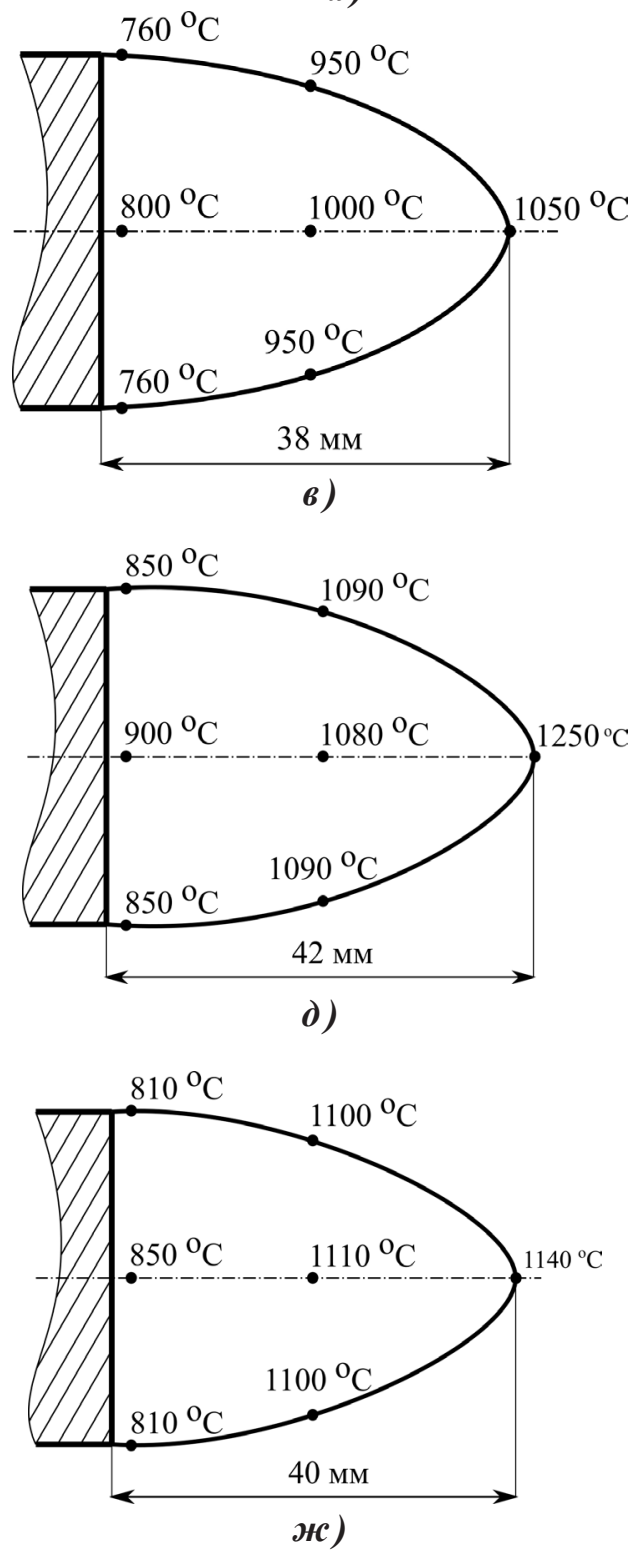

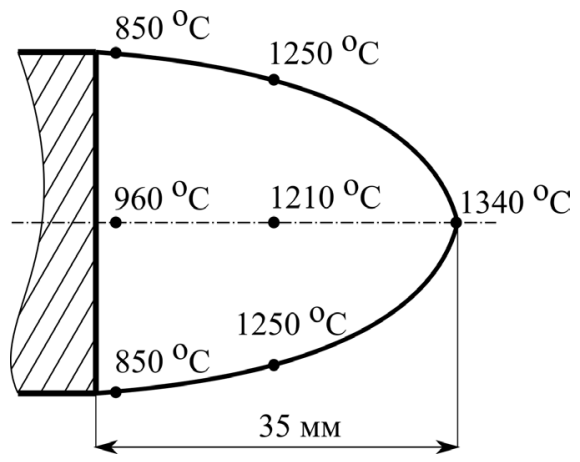

б)
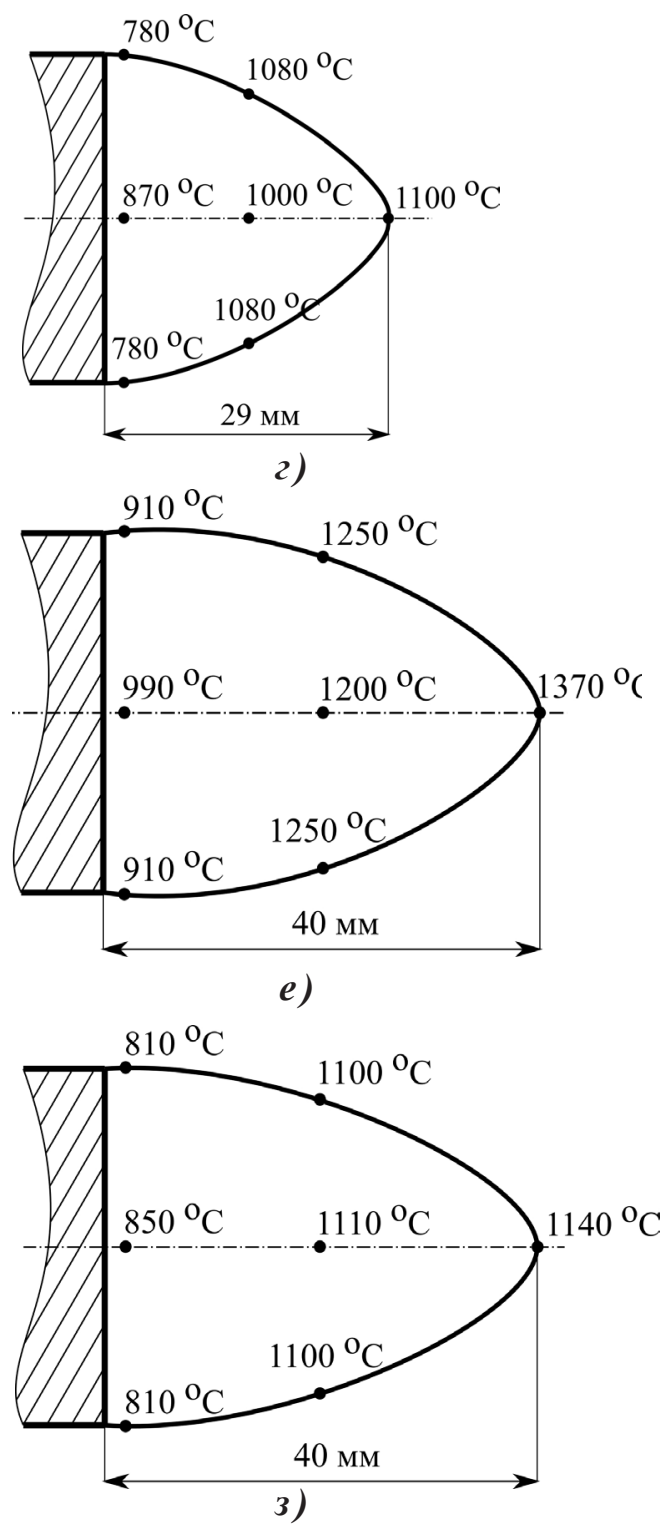

Рис. 2. Конфигурация границ зон обратных токов в ближнем следе цилиндрических стабилизаторов пламени и значения температур в характерных точках (1-5) этих зон для различных вариантов экспериментальных исследований; а, б, в, 2, д, е, же, з-варианты 1-8. 
стабилизатора. Иная картина наблюдалась для температурного перепада $\Delta t_{52}$ в поперечном сечении зоны обратных токов, отвечающем середине длины данной зоны. Здесь температурный перепад $\Delta t_{52}$ принимал для различных вариантов исследований как положительные, так и отрицательные значения. Иными словами температура на оси следа $t_{5}$ может либо превышать значения температуры $t_{2}$ на границе зоны обратных токов, либо быть меньше нее (см. данные табл. 2).

\section{Выводы}

В работе представлены результаты экспериментальных исследований, касающихся определения таких важных характеристик зон обратных токов в закормовых областях цилиндрических стабилизаторов пламени, как их конфигурация, размеры и температурный режим. При этом:

1. Установлены эффекты влияния на протяженность зон обратных токов относительного шага расположения газоподающих отверстий, скорости воздушного потока и коэффициента избытка воздуха. Показано, что увеличение указанного шага ввиду инжектирующего действия струй газа обусловливает сокращение протяженности данных зон. Отмечается в целом аналогичный характер влияния величины коэффициента избытка воздуха. Выявлено, что повышение скорости потока воздуха приводит к некоторому увеличению протяженности зон обратных токов.

2. Выполнен анализ особенностей температурных режимов зон обратных токов в ближнем следе стабилизаторов пламени. В частности, показано, что тенденция к повышению температуры в данной зоне с удалением от торцевой поверхности стабилизатора наиболее существенно проявляется вблизи этой поверхности. Установлено также, что с ростом относительного шага расположения газоподающих отверстий и скорости потока воздуха уровни максимальных температур в зоне обратных токов повышаются. При увеличении же коэффициента избытка воздуха наблюдается существенное падение данных температур.

\section{ЛИТЕРАТУРА}

1. Фиалко Н.М., Прокопов В.Г., Майсон Н.В., Шеренковский Ю.В., Иваненко Г.В., Абдулин М.З., Бутовский Л.С.,Ольховская Н.Н., Швецова Л.А., Дончак М.И. Особенности течения и смесеобразования в микрофакельных цилиндрических горелочных устройствах различной мощности // Научный вестник На-ционального университета биоресурсов и природопользования Украины Серия «Техника и энергетика АПК». - 2014. - 194/3. - C. $94-101$.

2. Фиалко Н.М., Шеренковский Ю.В., Майсон Н.В., Меранова Н.О., Абдулин М.З., Бутовский Л.С., Полозенко Н.П., Клищ, А.В., Стрижеус С.Н., Тимощенко А.Б. Математическое моделирование процессов течения и смесеобразования в цилиндрическом стабилизаторном горелочном устройстве // Восточно-европейский журнал передовых технологий. - 2014. - Т.3, №8(69). - С. 40 - 44.

3. Фиалко Н.М., Шеренковский Ю.В., Майсон Н.В., Меранова Н.О., Абдулин М.З., Бутовский Л.С., Полозенко Н.П., Клищ, А.В., Стрижеус С.Н., Тимощенко А.Б. Влияние пластинчатых турбулизаторов потока на ха-рактеристики течения и смесеобразования топлива и окислителя в цилиндрическом стабилизаторном го-релочном устройстве // Науковий вісник НЛТУ України. - 2014. - Вип. 24.06 - C. $114-121$.

4. Микулин Г. А., Любчик Г. Н. Аэродинамические характеристики и массообменные свойства трубчатых ин-тенсификаторов горения и стабилизаторов пламени // Энергетика: экономика, технология, экология. - 2004. - Т. 15, № 2. - С. 54 - 62.

5. Бутовский Л.С., Грановская Е.А., Фиалко Н.M Пусковые характеристики горелочных устройств стабили-заторного типа с подачей топлива в сносящий воздушный поток // Технологические системы. - 2010, № 4. - С. 32 - 37.

6. Раушенбах Б.В., Бельй С.А., Беспалов И.В., Бородачев В.Я., Вольнский М.С., Прудников А.Г. Физические основы рабочего процесса в камерах сгорания воздушно-реактивных двигателей. - М.: Машиностроение, 1964. - 526 с. 


\section{CHARACTERISTICS OF CIRCULATION FLOW IN NEAR WAKE OF CYLINDRICAL FLAME STABILIZERS}

Fialko N.M. ${ }^{1}$, Abdulin M.Z. ${ }^{2}$, Sherenkovskii Yu.V. ${ }^{1}$, Maison N.V. ${ }^{1}$

${ }^{1}$ Institute of Engineering Thermophysics of the National Academy of Sciences of Ukraine, Zhelyabova 2a, Kyiv, 03057, Ukraine

${ }^{2}$ National Technical University of Ukraine "Kyiv Polytechnic Institute", Prospect Peremohy 37, Kyiv, 03056, Ukraine

The present work is devoted to the experimental study of the regularities of flow and thermal conditions under propane-butane burning in cylindrical burner devices supplied to the air crossflow through the holes on the sides of the flame stabilizers. Particular attention is paid to temperature conditions, the configuration and size of the backflow zones in the near wake of these stabilizers. The data of influence on the characteristics of these zones such factors as the relative spacing of the gas injection holes, the air flow rate and the excess air ratio are presented.

References 6, tables 2, figures 2.

Key words: cylindrical flame stabilizer, backflow zone, temperature regime.

1. Fialko N.M., Prokopov V.G., Maison N.V., Sherenkovskii Yu.V., Ivanenko G.V., Abdulin M.Z., Butovskii L.S., Olkhovskaia N.N., Shvetsova L.A., Donchak M.I. Features of flow and mixing in microflame cylindrical burner de-vices of different capacities
// Nauchnyi vestnik Natsionalnogo universiteta bioresursov i prirodoispolzovaniia Ukrainy Seriia "Tehnika i energetika APK" . - 2014. - 194/3. - P. 94 -101 . (Rus.)

2. Fialko N.M., Sherenkovskii Yu.V., Maison N. V., Meranova N.O., Abdulin M.Z., Butovskii L.S., Polozenko N.P., Klishch A.V., Stryzheus S.N., Timoshchenko A.B. Numerical simulation of flow and mixing processes within cylin-drical stabiliser burner // Vostochno-evropeiskii zhyrnal peredovykh tekhnologii. - 2014. - V.3, №8(69). - P. 40 - 44. (Rus.)

3. Fialko N.M., Sherenkovskii Yu.V., Maison N. V., Meranova N.O., Abdulin M.Z., Butovskii L.S., Polozenko N.P., Klishch A.V., Stryzheus S.N., Timoshchenko A.B. Impact of flat flow energizers on flow characteristics and mixing of fuel and oxidizer within cylindrical stabilizer burner // Naukovyi visnyk NLTU Ukrainy. - 2014. - V. 24.06 - P. 114 - 121. (Rus.)

4. Mikulin G.A., Lubchik G.N. Aerodynamic characteristics and mass transfer properties of the tubular combustion intensificartors and flame stabilizers // Energetika: ekonomika, tekhnologiia, ekologiia. - 2004. - V. 15, № 2. - P. 54 - 62. (Rus.)

5. Butovskii L.S., Granovskaia E.A., Fialko N.M. Starting characteristics of stabilizer type burners with fuel injection into air cross flow // Tekhnologicheskie sistemy. - 2010, № 4. - P. 32 - 37. (Rus.)

6. Raushenbach B.V., Belyi S.A., Bespalov I.V., Borodachev V.Ya., Volynskii M.S., Prudnikov A.G. Physical basis of the working process in the combustion chambers of jet engines. - M.: Mashinostroenie, 1964. -526 pp. (Rus.)

Получено 06.04.2015 Received 06.04.2015 